\title{
NULLITY AND LOOP COMPLEMENTATION FOR DELTA-MATROIDS
}

\author{
ROBERT BRIJDER* AND HENDRIK JAN HOOGEBOOM ${ }^{\dagger}$
}

\begin{abstract}
We show that the symmetric difference distance measure for set systems, and more specifically for delta-matroids, corresponds to the notion of nullity for symmetric and skew-symmetric matrices. In particular, as graphs (i.e., symmetric matrices over $\mathrm{GF}(2)$ ) may be seen as a special class of delta-matroids, this distance measure generalizes the notion of nullity in this case. We characterize delta-matroids in terms of equicardinality of minimal sets with respect to inclusion (in addition we obtain similar characterizations for matroids). In this way, we find that, e.g., the delta-matroids obtained after loop complementation and after pivot on a single element together with the original delta-matroid fulfill the property that two of them have equal "null space" while the third has a larger dimension.
\end{abstract}

Key words. delta-matroid, local complementation, principal pivot transform, interlace polynomial, 4-regular graph

AMS subject classifications. 05B35, 05C50, 05C76, 15A09

1. Introduction. A set system is a tuple $M=(V, D)$ with $V$ a finite set, called the ground set, and $D$ a family of subsets of $V$. Set system $M$ is called proper if $D \neq \varnothing$. Let $X \subseteq V$. The pivot (also called twist) of $M$ on $X$, denoted by $M * X$, as $(V, D * X)$, where $D * X=\{Y \Delta X \mid Y \in D\}$ [4 (here $\Delta$ denotes symmetric difference). We denote by $\min (M)$ (and $\max (M)$, resp.) the set system with ground set $V$ consisting of the minimal (maximal, resp.) sets with respect to set inclusion of a set system $M$. A delta-matroid is a proper set system $M$ that satisfies the symmetric exchange axiom: For all $X, Y \in D$ and all $u \in X \Delta Y$, there is a $v \in X \Delta Y$ (possibly $v=u$ ) such that $X \Delta\{u, v\} \in D[4]$.

The main results of this paper are described below. We characterize first the notion of a delta-matroid.

TheOREm 4.2. Let $M$ be a proper set system. Then $M$ is a delta-matroid iff for each $X \subseteq V$, the sets in $\min (M * X)$ have equal cardinality.

We will almost exclusively work with this characterization rather than directly using the symmetric exchange axiom. Related to Theorem 4.2 we obtain novel characterizations of a matroid described by its bases and its independent sets. Below is the characterization of a matroid described by its independent sets.

Corollary 4.5. Let $M$ be a proper set system. Then $M$ is a matroid described by its independent sets iff both (1) for each $Y \in M$ and $Y^{\prime} \subseteq Y$, we have $Y^{\prime} \in M$, and (2) for each $X \subseteq V$, the sets in $\min (M * X)$ have equal cardinality.

Given a set system $M$ and a set $X$, the distance $d_{M}(X)=\min (\{|X \Delta Y| \mid Y \in$ $M\})$ is the minimal cardinality of the symmetric difference of $X$ and the sets in $M$. It turns out the distance behaves well under deletion of elements from the ground set provided we consider delta-matroids, cf. Theorem 5.2 .

For a set system $M=(V, D)$ and $v \in V$, we define pseudo-deletion of $M$ on $v$, denoted by $M \widehat{\Upsilon} v$, as $\left(V, D^{\prime}\right)$, where $D^{\prime}=\{X \cup\{v\} \mid X \in D, v \notin X\}$. Moreover, we define loop complementation of $M$ on $v$, denoted by $M+v$, as $\left(V, D^{\prime \prime}\right)$, where

\footnotetext{
${ }^{*}$ Hasselt University and Transnational University of Limburg, Belgium, robert.brijder@uhasselt.be

$\dagger$ Leiden Institute of Advanced Computer Science, Leiden University, The Netherlands, hoogeboom@liacs.nl
} 
$D^{\prime \prime}=D \Delta D^{\prime}=D \Delta\{X \cup\{v\} \mid X \in D, v \notin X\}$ [11]. Loop complementation is well motivated: it generalizes the loop complementation for graphs (this is recalled in Section 7). We derive the following property of delta-matroids in relation to pivot and loop complementation.

TheOrem [5.6. Let $M$ be a delta-matroid, and $v \in V$ such that $M+v$ is a delta-matroid. Then $\max (M), \max (M * v)$, and $\max (M+v)$ are matroids (described by their bases) such that precisely two of the three are equal, to say $M_{1}$. Moreover, the rank of the third $M_{2}$ is one smaller than the rank of $M_{1}$ and $M_{2} \widehat{\backslash v}=M_{1}$.

We also formulate a "min counterpart" of Theorem [5.6. cf. Theorem [5.5. which involves the dual pivot operation instead of the loop complementation operation.

Given a $V \times V$ matrix $A$ (the columns and rows of $A$ are indexed by finite set $V$ ), we denote by $A[X]$ the principal submatrix of $A$ induced by $X$ and we define the set system $\mathcal{M}_{A}=\left(V, D_{A}\right)$ with $D_{A}=\{X \subseteq V \mid A[X]$ is nonsingular $\}$. If $A$ is skew-symmetric (i.e., $A^{T}=-A$ where $A^{T}$ denotes the transpose of $A$ ) or symmetric, then $\mathcal{M}_{A}$ is a delta-matroid [4. We show that nullity of a principal submatrix $A[X]$ corresponds to distance in the associated delta-matroid $\mathcal{M}_{A}$. This is the main motivation for considering distance, as it allows us to carry over results of distances in delta-matroids to nullity values of symmetric or skew-symmetric matrices and graphs in particular.

Theorem 6.2. Let $A$ be a $V \times V$ symmetric or skew-symmetric matrix (over some field). Then $d_{\mathcal{M}_{A}}(X)=n(A[X])$ for each $X \subseteq V$.

It is known that $\mathcal{M}_{A} * X$, for any $V \times V$ matrix $A$ and any set $X$ in $\mathcal{M}_{A}$, is equal to $\mathcal{M}_{A * X}$ where $A * X$ is the principal pivot transform of $X$ on $A$ (see [24, 23] for the definition of this notion). Hence there is a close connection between the linear algebra of principal pivot transform and the combinatorics of pivot on set systems.

The delta-matroid $\mathcal{M}_{A}$ for a symmetric or skew-symmetric matrix $A$ is especially interesting over the binary field $\mathbb{F}_{2}$ (note that the notions of skew-symmetric and symmetric coincide over $\mathbb{F}_{2}$ ), i.e., in the case where $A$ is a graph (where loops are allowed). In this case $\mathcal{M}_{A}$ retains all information of $A$ - hence $\mathcal{M}_{A}$ is a representation of the graph $A$. It turns out that for a graph the null space (kernel) of its adjacency matrix is determined by the set of maximal elements in the associated delta-matroid. In this way we obtain the following result (we associate a graph $G$ by its adjacency matrix $A(G)$ ). For a graph and vertex $v, G+v$ denotes loop complementation (the existence of a loop on $v$ is complemented), and if $v$ is a looped vertex, then $G * v$ denotes principal pivot transform on $v$, which over $\mathbb{F}_{2}$ is local complementation (the subgraph of the neighbourhood of $v$ is complemented). As usual, we identify vectors indexed by $V$ over $\mathbb{F}_{2}$ by subsets of $V$.

TheOREm 7.1. Let $G$ be a graph having a looped vertex $v$. Then $\operatorname{ker}(G)$, $\operatorname{ker}(G * v)$, and $\operatorname{ker}(G+v)$ are such that precisely two of the three are equal, to say $K_{1}$, and the third, $K_{2}$, is such that $\operatorname{dim}\left(K_{2}\right)=\operatorname{dim}\left(K_{1}\right)+1$ and $K_{1}=\left\{X \in K_{2} \mid v \notin X\right\}$.

This result is related to [22, Lemma 23] (there a graph different from $G * v$ is considered) and [3, Theorem (9.4)], and it can be seen as an extension of [1, Lemma 2]. In case $G$ is a circle graph, Theorem 7.1 is applicable to the theory of closed walks in 4-regular graphs, see e.g. [21.

It is known from [11] that the family of delta-matroids is not closed under loop complementation. We show in Section 8 that the family of binary delta-matroids is closed under pivot, loop complementation and deletion of elements from the ground set.

The results given in this paper are crucial in a subsequent paper on interlace 
polynomials of delta-matroids $[9]$.

2. Pivot and Loop Complementation on Set Systems. First we fix basic notions and terminology. We denote the field consisting of two elements by $\mathbb{F}_{2}$. In this field addition and multiplication are equal to the logical exclusive-or and logical conjunction, which are denoted by $\oplus$ and $\wedge$ respectively. By carrying over $\oplus$ to sets, we obtain the symmetric difference operator $\Delta$. Hence for sets $A, B \subseteq V$ and $x \in V$, $x \in A \Delta B$ iff $(x \in A) \oplus(x \in B)$.

A set system (over $V$ ) is a tuple $M=(V, D)$ with $V$ a finite set, called the ground set, and $D$ a family of subsets of $V$. Let $X \subseteq V$. We define $M[X]=\left(X, D^{\prime}\right)$ where $D^{\prime}=\{Y \in D \mid Y \subseteq X\}$, and define $M \backslash X=M[V \backslash X]$. Set system $M$ is called proper if $D \neq \varnothing$. Let $\min (D)(\max (D)$, resp.) be the family of minimal (maximal, resp.) sets in $D$ with respect to set inclusion, and let $\min (M)=(V, \min (D))(\max (M)=$ $(V, \max (D))$, resp.) be the corresponding set systems. Also, we denote the family of minimal sets with respect to cardinality by $\operatorname{minc}(D)$, i.e., $X \in \operatorname{minc}(D)$ iff $X \in D$ and $|X| \leq|Y|$ for all $Y \in D$. We let $\operatorname{minc}(M)=(V, \operatorname{minc}(D))$ be the corresponding set system. Similarly, we define $\operatorname{maxc}(M)=(V, \operatorname{maxc}(D))$. Note: we will also use $\min (E)$ and $\max (E)$ for a finite set $E$ of integers, to denote the smallest and largest, resp., integer in $E$. We simply write $Y \in M$ to denote $Y \in D$, and for set system $M^{\prime}=\left(V, D^{\prime}\right), M \subseteq M^{\prime}$ if $D \subseteq D^{\prime}$. We also often write $V$ to denote the ground set of the set system under consideration. A set system $M$ is called equicardinal if for all $X_{1}, X_{2} \in M,\left|X_{1}\right|=\left|X_{2}\right|$.

Let $M=(V, D)$ be a set system. We define, for $X \subseteq V$, pivot of $M$ on $X$, denoted by $M * X$, as $(V, D * X)$, where $D * X=\{Y \Delta X \mid Y \in D\}$. The pivot operation (often called twist in the literature) is often denoted by $M \Delta X$ instead of $M * X$ (see, e.g., (4)). However, as $D * X$ is of course in general different from $D \Delta X$, to avoid confusion, we use $*$ for pivot. We define, for $X \subseteq V$, loop complementation of $M$ on $X$ (the motivation for this name is from graphs, see Section [7), denoted by $M+X$, as $\left(V, D^{\prime}\right)$, where $Y \in D^{\prime}$ iff $|\{Z \in M \mid Y \backslash X \subseteq Z \subseteq Y\}|$ is odd [1]. In particular, if $X=\{v\}$ is a singleton, then $D^{\prime}=D \Delta\{Z \cup\{v\} \mid Z \in D, v \notin Z\}$.

For notational convenience we often omit the "braces" for singletons $\{v\}$, and write, e.g., $M+v, M * v$, and $M \backslash v$. Loop complementation and pivot belong to a class of operations called vertex flips, cf. [11. Deletion $M \backslash u$ is also a vertex flip operation (modulo a, for this purpose irrelevant, difference in ground set). To simplify notation, we assume left associativity of the vertex flips, and write, e.g., $M * u+v$ to denote $(M * u)+v$. Vertex flips turn out commute on different elements. Therefore, if $u, v \in V$ and $u \neq v$, then, e.g., $M+u \backslash v=M \backslash v+u, M * u \backslash v=M \backslash v * u$, $M * u+v=M+v * u$, and $M+u+v=M+v+u$. Moreover, it is easy to verify that $M+u \backslash u=M \backslash u$.

It has been shown in 11 that pivot $* u$ and loop complementation $+u$ on a common element $u \in V$ are involutions (i.e., of order 2) that generate a group isomorphic to $S_{3}$, the group of permutations on 3 elements. In particular $+u * u+u=$ $* u+u * u$ is the third involution, called the dual pivot, and is denoted by $\bar{*}$. We have, e.g., $+u * u=\bar{*} u+u=* u \bar{*} u$ and $* u+u=+u \bar{*} u=\bar{*} u * u$ for $u \in V$ (these are the two operations of order 3 ). The six operations (including the identity operation) are called invertible vertex flips.

It turns out that, for $X \subseteq V, M \mp X=M+X * X+X$ is equal to $\left(V, D^{\prime}\right)$, where $Y \in D^{\prime}$ iff $|\{Z \in M \mid Y \subseteq Z \subseteq Y \cup X\}|$ is odd. In particular, if $X=\{v\}$ is a singleton, then $D^{\prime}=D \Delta\{Z \backslash\{v\} \mid Z \in D, v \in Z\}$. Equivalently, for $Y \subseteq V$, if $v \in Y$, then $Y \in M \bar{*} v$ iff $Y \in M$, and if $v \notin Y$, then $Y \in M \bar{*} v$ iff $(Y \in M) \oplus(Y \Delta\{v\} \in M)$. 
Finally, it is observed in [11] that $\min (M)=\min (M+X)$. Since $\min (M)=$ $\max (M * V) * V$, we have similarly $\max (M)=\max (M \bar{*} X)$.

We will often use the results of this section without explicit mention.

3. Distance in Set Systems. Let $M$ be a proper set system. For $X \subseteq V$, we define $d_{M}(X)=\min (\{|X \Delta Y| \mid Y \in M\})$. Hence, $d_{M}(X)$ is the smallest distance between $X$ and the sets in $M$, where the distance between two sets is measured as the number of elements in the symmetric difference. We will study some properties of this natural notion, and in particular we investigate the relation between the values $d_{M \rho}(X)$ for different invertible vertex flips $\rho$ on a fixed element $v$.

We set $d_{M}=d_{M}(\varnothing)$, the cardinality of a smallest set in $M$.

Lemma 3.1. Let $M$ be a proper set system. Then $d_{M * Z}(X)=d_{M}(X \Delta Z)$ for all $X, Z \subseteq V$.

Proof. $\quad d_{M * Z}(X)=\min (\{|X \Delta Y| \mid Y \in M * Z\})=\min (\{|X \Delta(Y \Delta Z)| \mid Y \in$ $M\})=\min (\{|(X \Delta Z) \Delta Y| \mid Y \in M\})=d_{M}(X \Delta Z)$.

This basic fact is mainly used to reduce (without loss of generality) results concerning distance from $X \subseteq V$ in set systems to distance from the empty set, i.e., the cardinality of the smallest set in $M: d_{M}(X)=d_{M * X}(\varnothing)=d_{M * X}$.

As $\min (M)=\min (M+v)$ we infer that the six different invertible vertex flips on $v$ result in at most three different values: $d_{M}=d_{M+v}, d_{M * v}=d_{M * v+v}$, and $d_{M \bar{*} v}=d_{M+v * v}$. By Lemma 3.1 this can be extended to distance between an arbitrary $X \subseteq V$ (instead of $\varnothing$ ) and $M$. When $v \notin X$ then the three equalities above hold essentially unchanged for distance from $X$ since vertex flip $\rho$ on $v$ and pivot on $X$ commute: $d_{M \rho}(X)=d_{M \rho * X}(\varnothing)=d_{M * X \rho}$. However, when $v \in X$ this commutation no longer holds, and we have to reconsider the equalities. Writing $X^{\prime}=X \Delta\{v\}$ and $M^{\prime}=M * X^{\prime}$ we have then

- $d_{M}(X)=d_{M^{\prime} * v}=d_{M^{\prime} * v+v}=d_{M \bar{*} v}(X)$

- $d_{M * v}(X)=d_{M^{\prime}}=d_{M^{\prime}+v}=d_{M+v * v}(X)$

- $d_{M+v}(X)=d_{M^{\prime}+v * v}=d_{M^{\prime} \text { * } v}=d_{M * v+v}(X)$

One easily argues that applying an invertible vertex flip changes $d_{M}$ by at most one.

Lemma 3.2. Let $M$ be a proper set system. If $\rho$ is an invertible vertex flip of $M$ on $v \in V$, then (1) $\left|d_{M}-d_{M \rho}\right| \leq 1$ and (2) $d_{M \rho} \in\{m, m+1\}$ with $m=$ $\min \left(\left\{d_{M}, d_{M * v}\right\}\right)$.

Proof. Proof of (1). By the above, we need only to verify the cases $\rho=* v$ and $\rho=\bar{*} v$. By the definitions of pivot and dual pivot, for any pair of sets $Z, Z \Delta\{v\} \subseteq V$, at least one of this pair is in $M$ iff at least one of this pair is in $M \rho$. Hence the smallest cardinality of a set in $M$ cannot differ by more than one from the smallest cardinality of a set in $M \rho$.

Proof of (2). By (1), the result is valid for $d_{M}$ and $d_{M * v}$, and it suffices to show that $d_{M \bar{*} v} \geq m$. The argument we use works for any invertible vertex flip $\rho$. Let $Z \in \operatorname{minc}(M \rho)$, i.e., $Z \in M \rho$ such that $|Z|=d_{M \rho}$. We have $Z \in M$ or $Z \Delta\{v\} \in M$ (or both). If $Z \in M$, then $d_{M \rho}=|Z| \geq d_{M} \geq m$. If $Z \Delta\{v\} \in M$, then $Z \in M *\{v\}$ and $d_{M \rho}=|Z| \geq d_{M * v} \geq m$. Hence in both cases we have $d_{M \rho} \geq m$.

By Lemma 3.2 the three values $d_{M}, d_{M * v}$, and $d_{M \star v}$ cannot be all different. As $d_{M}(X \Delta\{v\})=d_{M *\{v\}}(X)$ we also have, for $v \in V,\left|d_{M}(X)-d_{M}(X \Delta\{v\})\right| \leq 1$.

We obtain now a result for $M \bar{*} v$ assuming $d_{M} \neq d_{M * v}$. 
TheOREm 3.3. Let $M$ be a proper set system, and $v \in V$. We have

$$
\operatorname{minc}(M \bar{*} v)=\left\{\begin{array}{ll}
\operatorname{minc}(M) & d_{M}<d_{M * v} \\
\operatorname{minc}(M * v) & d_{M * v}<d_{M}
\end{array} .\right.
$$

In either case, the elements of $\operatorname{minc}(M \bar{*} v)$ do not contain $v$. In particular, if $d_{M} \neq d_{M * v}$, then $d_{M \bar{*} v}=m$ with $m=\min \left(\left\{d_{M}, d_{M * v}\right\}\right)$.

Proof. We may assume without loss of generality that $d_{M}<d_{M * v}$. Indeed, if $d_{M}>d_{M * v}$, then consider $M^{\prime}=M * v$. We have $\operatorname{minc}(M \bar{*} v)=\operatorname{minc}\left(M^{\prime} * v \bar{*} v\right)=$ $\operatorname{minc}\left(M^{\prime} \bar{*} v+v\right)=\operatorname{minc}\left(M^{\prime} \bar{*} v\right)$ and $d_{M^{\prime}}<d_{M^{\prime} * v}$.

Assume therefore that $d_{M}<d_{M * v}$. Let $Z \in \operatorname{minc}(M)$. Note that $v \notin Z$ as otherwise $Z \Delta\{v\}$ would be a smaller set in $M * v$. Moreover $Z \notin M * v$, as otherwise $d_{M * v} \leq d_{M}$. By the definition of dual pivot, $v \notin Z$ implies that $Z \in M \bar{*} v$ iff exactly one of $Z$ and $Z \cup\{v\}$ is in $M$. Hence $Z \in M \mp v$. By Lemma 3.2, $d_{M \mp v} \geq d_{M}$ and so $d_{M \bar{*} v}=d_{M}$ and $Z \in \operatorname{minc}(M \bar{*} v)$.

Consider now $M^{\prime \prime}=M \bar{*} v$. We have seen that $d_{M \bar{*} v}=d_{M}$, and so $d_{M^{\prime \prime}}=d_{M}<$ $d_{M * v}=d_{M * v+v}=d_{M * v * v}=d_{M^{\prime \prime * v}}$. By the first part of this proof we have that $Z \in \operatorname{minc}\left(M^{\prime \prime}\right)=\operatorname{minc}(M \bar{*} v)$ implies $Z \in \operatorname{minc}\left(M^{\prime \prime} \bar{*} v\right)=\operatorname{minc}(M)$. Consequently, we obtain $\operatorname{minc}(M \bar{*} v)=\operatorname{minc}(M)$. $\square$

From this result we see that the values of $d_{M}, d_{M * v}$, and $d_{M \text { * } v}$ are either (1) all equal or (2) of the form $m, m, m+1$ (in some order). We show in Section 5 that for delta-matroids only the latter case occurs.

EXAmple 3.1. Let $V=\{a, b, c\}$, and let $M$ be the set system $(V,\{\{a\},\{b, c\}\})$. We have $M * b=(V,\{\{a, b\},\{c\}\})$ and $M \mp b=(V,\{\{a\},\{c\},\{b, c\}\})$. Hence $d_{M}=$ $d_{M * b}=d_{M * b}=1$.

4. A Characterization of Delta-Matroids. By Lemma 3.1 $d_{M}(X)=d_{M * X}$ is the minimal cardinality of the sets in $M * X$. As a consequence, each set in $M * X$ of cardinality $d_{M}(X)$ belongs to $\min (M * X)$, but the converse does not necessarily hold, i.e., the inclusion $\operatorname{minc}(M * X) \subseteq \min (M * X)$ may not be an equality. We consider now set systems with the property that the converse does hold: for each $X \subseteq V$, the sets in $\min (M * X)$ are all of equal cardinality (or equivalently, $\operatorname{minc}(M * X)=\min (M * X))$.

Definition 4.1. A proper set system $M$ over $V$ is called isodistant if, for each $X \subseteq V, \min (M * X)$ is equicardinal.

Thus for isodistant $M$, the common cardinality of the sets $\operatorname{in} \min (M * X)$ is equal to $d_{M}(X)$. As we have noted this also holds the other way around, and so the minimal sets in $M * X$ are characterized by their cardinality.

Clearly, the isodistant property of set systems is invariant under pivot: if set system $M$ is isodistant, then $M * X$ is isodistant for each $X \subseteq V$. Due to the duality $\min (M) * V=\max (M * V)$, one easily verifies that $M$ is isodistant iff for each $X \subseteq V$, $\max (M * X)$ is equicardinal. In that case the sets in $\max (M * X)$ are all of cardinality equal to $|V|-d_{M}(V \backslash X)$.

A delta-matroid is a proper set system $M$ that satisfies the symmetric exchange axiom: For all $X, Y \in M$ and all $u \in X \Delta Y$, either $X \Delta\{u\} \in M$ or there is a $v \in X \Delta Y$ with $v \neq u$ such that $X \Delta\{u, v\} \in M$ [4]. The notion of delta-matroid is equivalent to the notion of Lagrangian matroid [2, Section 6]. If we assume a matroid $M$ is described by a tuple $(V, B)$ where $B$ is the set of bases of $M$, then it is shown in [6. Proposition 3] that a matroid $M$ is precisely a equicardinal delta-matroid (the result essentially follows from [12, Theorem 1]). It is stated in [7, Property 4.1] that a set system $M$ is a delta-matroid iff $\max (M * X)$ is a matroid (described by its bases) for every $X \subseteq V$. Consequently, every delta-matroid is isodistant. 
We now show that, surprisingly, the converse holds. Hence, the notions of deltamatroid and isodistance are equivalent, i.e., without assuming the matroid structure of the maximal or minimal elements.

TheOREM 4.2. Let $M$ be a proper set system. Then $M$ is a delta-matroid iff $M$ is isodistant.

Proof. Assume first that $M$ is isodistant. Let $X, Y \in M$ and $u \in X \Delta Y$. We need to show that either $X \Delta\{u\} \in M$ or there is a $v \in X \Delta Y$ with $v \neq u$ such that $X \Delta\{u, v\} \in M$. Consider $M^{\prime}=M *(X \Delta\{u\})$. If $\varnothing \in M^{\prime}$, then $X \Delta\{u\} \in M$ and we are done. Assume $\varnothing \notin M^{\prime}$. We have $\{u\} \in M^{\prime}$ and $Z=Y \Delta(X \Delta\{u\}) \in M^{\prime}$. As $M$ is isodistant, so is $M^{\prime}$ and thus $\{v\} \in M^{\prime}$ for some $v \in Z$. As $u \notin Z, u \neq v$. Therefore, $X \Delta\{u, v\} \in M$ and we are done.

The forward implication, i.e., the fact that the maximal elements of a deltamatroid are of equal cardinality, follows from [7, Property 4.1] (stated above) or [13, Lemma 6]. $\mathrm{c}$

By restricting to equicardinal set systems we obtain the following corollary.

Corollary 4.3. Let $M$ be a proper set system. Then $M$ is a matroid described by its bases iff both (1) $M$ is equicardinal, and (2) for each $X \subseteq V, \min (M * X)$ is equicardinal.

Although the characterization of a matroid in Corollary 4.3 is novel, we can link it to a well-known characterization of matroids $M$ given below, where $M$ is described by its independent sets. This characterization can be found, e.g., in [20, Exercise 1.1.3] and in [25, Section 1.5].

Proposition 4.4. Let $M$ be a proper set system. Then $M$ is a matroid described by its independent sets iff both (1) for each $Y \in M$ and $Y^{\prime} \subseteq Y$, we have $Y^{\prime} \in M$, and (2) for each $X \subseteq V, \max (M[X])$ is equicardinal.

The second property of Proposition 4.4 is known as the cardinality property.

Inspired by Proposition 4.4 and Corollary 4.3 we obtain the following result, which from appearance may be thought of as the "analog" of Corollary 4.3 where the matroid is described by its independent sets (it appears that there is no obvious "analog" of Proposition 4.4 for matroids described by its bases).

Corollary 4.5. Let $M$ be a proper set system. Then $M$ is a matroid described by its independent sets iff both (1) for each $Y \in M$ and $Y^{\prime} \subseteq Y$, we have $Y^{\prime} \in M$, and (2) for each $X \subseteq V, \min (M * X)$ is equicardinal.

Proof. Let $M$ be a proper set system such that condition (1) holds. Let $X \subseteq V$, and let $Z \in \min (M * X)$. Then $Z \Delta X \in M$. If $v \in Z \backslash X$, then $(Z \backslash\{v\}) \Delta X \subset Z \Delta X$ and $(Z \backslash\{v\}) \Delta X \in M$ by condition (1), contradicting the minimality of $Z$. Therefore $Z \subseteq X$. Consequently, $Z \Delta X \subseteq X$. Hence $\min (M * X)=\min (M[X] * X)$.

As $X$ is the ground set of $M[X]$, we have $\min (M[X] * X)=\max (M[X]) * X$. Again, as $X$ is the ground set of $M[X], \max (M[X]) * X$ is equicardinal iff $\max (M[X])$ is equicardinal. The result follows now by Proposition 4.4. $\mathrm{\square}$

Note that, again, $\min (M * X)$ in Corollary 4.5 may equivalently be replaced by $\max (M * X)$. Also note that while the second condition of Corollary 4.3 and of Corollary 4.5 are identical, they concern (in general) very different set systems. Indeed, if $M$ is a matroid described by its independent sets, then $\max (M)$ is the corresponding matroid described by its bases.

From now on, we prefer the term delta-matroid instead of the equivalent notion of isodistant set system, as the former is well known. However, the results in this paper do not (directly) use the definition of delta-matroid; we use only the property of isodistance. 
5. Distance in Delta-Matroids. We reconsider the distance function $d_{M}$, but now restricted to delta-matroids $M$ rather than set systems in general.

We may now characterize delta-matroids through distance and deletion.

TheOREm 5.1. Let $M$ be a proper set system. Then $M$ is a delta-matroid iff $d_{M * Y}=d_{M * Y[X]}$ for all $X, Y \subseteq V$ with $M * Y[X]$ proper.

Proof. We first show the forward implication. It is easy to see that $\min (M[X]) \subseteq$ $\min (M)$ for any set system $M$ and $X \subseteq V$ with $M[X]$ proper. Hence if $M$ is a deltamatroid, then $d_{M}=d_{M[X]}$, and similarly for $M * Y$ for all $Y \subseteq V$. To show the reverse implication, assume $M$ is not a delta-matroid, and let $Z_{1}, Z_{2} \in \min (M * Y)$ with $\left|Z_{1}\right|<\left|Z_{2}\right|$. We have that $M * Y\left[Z_{2}\right]$ consists only of $Z_{2}$ and therefore $d_{M * Y\left[Z_{2}\right]}=\left|Z_{2}\right|$. However, $d_{M * Y} \leq\left|Z_{1}\right|$ - a contradiction. $\square$

It follows from Theorem 5.1 that the distance function $d_{M}$ behaves well under removal of elements from the ground set $V$.

Theorem 5.2. Let $M$ be a delta-matroid, and $X \subseteq V$. If $M[X]$ is proper, then $d_{M[X]}(Y)=d_{M}(Y)$ for all $Y \subseteq X$.

Proof. We have $d_{M}(Y)=d_{M * Y}$ and $d_{M[X]}(Y)=d_{M[X] * Y}=d_{M * Y[X]}$ where in the last equality we use $Y \subseteq X$ and the commutation of vertex flips. The result holds by Theorem 5.1 .

In particular, by Theorem 5.2, $d_{M}(X)=d_{M[X]}(X)$ for all $X \subseteq V$ where $M[X]$ is proper, hence $d_{M}(X)=\min (\{|X \backslash Y| \mid Y \in M, Y \subseteq X\})$.

The property of delta-matroids shown in Theorem 5.2 is important in a subsequent study of interlace polynomials on delta-matroids 9 . Of course, Theorem 5.2 does not hold for set systems in general. Indeed, it is easy to verify that set system $M=(V,\{\varnothing, V\})$ is not a delta-matroid for $|V| \geq 3$. Take $|V|=3$. We have, for $u \in V, M \backslash u=(V \backslash\{u\},\{\varnothing\})$ and therefore $2=d_{M \backslash u}(V \backslash\{u\}) \neq d_{M}(V \backslash\{u\})=1$. It is also easy to verify that the property of Theorem 5.2 does not characterize deltamatroids like in Theorem 5.1 (take, e.g., $M=(\{a, b, c\},\{\varnothing,\{a\},\{b, c\}\}))$.

By Theorem 3.3. for arbitrary set systems the value of $d_{M \bar{*} v}$ is the minimum of $d_{M}$ and $d_{M * v}$ when these two values differ. However, the value of $d_{M \bar{*} v}$ could not be fixed when $d_{M}$ equals $d_{M * v}$. This changes when $M$ is a delta-matroid.

Lemma 5.3. Let $M$ be a delta-matroid, and $v \in V$ such that $d_{M}=d_{M * v}$. Then (1) no set in $\min (M)$ contains $v,(2) \min (M)=\min (M * v)$, and (3) $d_{M \bar{*} v}=d_{M}+1$.

Proof. Let $m=d_{M}=d_{M * v}$. (1) Let $Y \in \min (M)$. Then $Y \Delta\{v\} \in M * v$. As $|Y| \leq|Y \Delta\{v\}|$, we have $v \notin Y$.

(2) Let $Y \in \min (M)$. As $Y \cup\{v\} \in M * v$, there must be a $Y^{\prime} \in \min (M * v)$ of cardinality $m$ with $Y^{\prime} \subseteq Y$. If $Y^{\prime}=Y \cup\{v\} \backslash\{w\}$ with $v \neq w$, then $Y^{\prime} \backslash\{w\} \in$ $\min (M)$ while this set is smaller than $m-$ a contradiction. Hence $v=w$, and $Y=Y^{\prime} \in \min (M * v)$. Therefore, $\min (M) \subseteq \min (M * v)$. By symmetry we obtain the other inclusion.

(3) By Lemma 3.2. $d_{M \bar{*} v} \in\{m, m+1\}$, so it suffices to prove that there are no sets in $M \bar{*} v$ that have cardinality $m$. Thus assume $Z \in M \bar{*} v$ and $|Z|=m$. By the definition of dual pivot, either $Z \in M$ (case $v \in Z$ ) or $Z$ in exactly one of $M$ and $M * v$ (case $v \notin Z$ ). In the former case we have $Z \in \min (M)$ while $v \in Z$, contradicting (1). The latter case contradicts with (2).

It is observed in [1] that both loop complementation and dual pivot do not (in general) retain the property of being a delta-matroid. For example, for deltamatroid $M=\left(V, 2^{V} \backslash\{V\}\right)$ with $V=\{1,2,3\}, M \bar{*} 1$ is not a delta-matroid. In fact, $\min (M \bar{*} 1)=(V,\{\{1\},\{2,3\}\})$ is not even equicardinal. The next result shows that 
$\min (M \bar{*} v)$ is equicardinal for a delta-matroid $M$ when $d_{M} \neq d_{M * v}$.

Lemma 5.4. Let $M$ be a delta-matroid, and $v \in V$. If $d_{M} \neq d_{M * v}$, then $\min (M \bar{*}$ v) is equicardinal.

Proof. Assume first that $d_{M}<d_{M * v}$. By Theorem 3.3, $d_{M}=d_{M \bar{*} v}$, and $\min (M)=\operatorname{minc}(M \bar{*} v) \subseteq \min (M \bar{*} v)$. Let $Z \in \min (M \bar{*} v)$. By definition of dual pivot, $Z \in M$ or $Z \Delta\{v\} \in M$ (or both). Hence, as no set in $\min (M)$ contains $v$, there is a $Y \in \min (M)$ with $Y \subseteq Z \backslash\{v\}$. Again, $Y \in \min (M)$ implies $Y \in \min (M \bar{*} v)$ and so $Y=Z$.

The other case, $d_{M}>d_{M * v}$, follows by symmetry (consider the delta-matroid $M^{\prime}=M * v$ similar as in the proof of Theorem 3.3).

Let $M=(V, D)$ be a set system and $v \in V$. We define pseudo-deletion of $M$ on $v$, denoted by $M \widehat{\backslash v}$, as $M \widehat{\backslash v}=(V, D \backslash v * v)$. Similarly, we define pseudo-contraction of $M$ on $v$, denoted by $M \widehat{/ v}$, as $(V, D * v \backslash v)$. Note that the ground sets for both pseudodeletion and pseudo-contraction remain unchanged. Also note that $M * v \widehat{\backslash v}=M \widehat{/ v} * v$.

The definitions of pseudo-deletion and pseudo-contraction are motivated by matroids as follows. Recall that for a matroid $M$ described by its bases and $v \in V$, $M \backslash v$ and $M * v \backslash v$ are the matroid operations of deletion (if $v$ is not a coloop) and contraction (if $v$ is not a loop), denoted by $M \backslash v$ and $M / v$, respectively. It is easy to see that then pseudo-deletion is adding $v$ as a coloop to $M \backslash v$ and pseudo-contraction is adding $v$ as a loop to $M / v$ [3]. In this way, we regard pseudo-deletion and pseudocontraction as matroid operations as well. Pseudo-deletion and pseudo-contraction take the following form if a matroid is described by its circuits. If $M^{\prime}=(V, \mathcal{C})$ is the circuit description of $M$, then $M^{\prime} \widehat{\backslash v}=(V, \mathcal{C} \backslash v)$ and $M^{\prime} \widehat{/} v=(V,(\mathcal{C} / v) \cup\{\{v\}\})$.

We are now ready to formulate the announced $m, m, m+1$ result for deltamatroids.

Theorem 5.5. Let $M$ be a delta-matroid, and $v \in V$. Then the equicardinal set systems $\min (M), \min (M * v)$, and $\operatorname{minc}(M \bar{*} v)$ are such that precisely two of the three are equal, to say $M_{1}$. Moreover, the third $M_{2}$ is such that $d_{M_{2}}=d_{M_{1}}+1$ and $M_{2} \widehat{/ v}=M_{1}$.

In particular, the values of $d_{M}, d_{M * v}$, and $d_{M \bar{*}}$ are such that precisely two of the three are equal, to say $m$, and the third is equal to $m+1$.

Proof. (i) The case $d_{M}=d_{M * v}$ follows from Lemma 5.3 except for the equality $M_{2} \widehat{/ v}=M_{1}$. Let $Z \in M_{1}=\min (M * v)$. By Lemma 5.3, $v \notin Z$. We have $Z \Delta\{v\} \in M$ and $v \in Z \Delta\{v\}$, and therefore $Z \Delta\{v\} \in M \bar{*} v$ and $|Z \Delta\{v\}|=d_{M \bar{*} v}$. Hence, $Z \Delta\{v\} \in \operatorname{minc}(M \bar{*} v)=M_{2}$. Conversely, let $Z \in \operatorname{minc}(M \bar{*} v)=M_{2}$ with $v \in Z$. Then $Z \in M$. Hence $Z \backslash\{v\} \in M * v$. Since $d_{M_{2}}=d_{M_{1}}+1, Z \backslash\{v\} \in \min (M * v)=M_{1}$. (ii) Consider $d_{M}<d_{M * v}$, hence $d_{M}+1=d_{M * v}$. By Theorem 3.3 we know that $\operatorname{minc}(M \bar{*} v)=\min (M)$. If $Z \in \min (M)$, then $Z \Delta v \in M * v$ is minimal by cardinality and so $\min (M) * v \subseteq \min (M * v)$. Conversely, if $Z \in \min (M * v)$ and $v \in Z$, then $Z \backslash\{v\} \in \min (M)$ as $d_{M_{2}}=d_{M_{1}}+1$. (iii) The case $d_{M * v}<d_{M}$ holds by symmetry. $\mathbf{\square}$

Theorem 5.5 is related to Theorem (9.4) of [3], which deals fundamentally with binary matroids. In fact, Theorem 5.5 may be seen as a generalisation of Theorem (9.4) of [3], cf. Theorem 7.1

Note that the cardinality of the sets in $M_{2}$ is exactly one larger than the cardinality of the sets in $M_{1}$. Also note that $\min (M)$ and $\min (M * v)$ are matroids and with ranks $d_{M}$ and $d_{M * v}$, respectively. Lemma 5.4 shows that if $d_{M} \neq d_{M * v}$, then $\operatorname{minc}(M \bar{*} v)=$ $\min (M \bar{*} v)$ is also a matroid with rank $d_{M \bar{*} v}$. Of course, if $M \bar{*} v$ is a delta-matroid, then $\operatorname{minc}(M \bar{*} v)=\min (M \bar{*} v)$ also holds. 
Let $X \subseteq V$. By Theorem 5.5, if $v \notin X$, then the values of $d_{M}(X), d_{M * v}(X)$, and $d_{M \bar{*} v}(X)$ are such that precisely two of the three are equal, to say $m$, and the third is equal to $m+1$. Also, if $v \in X$, then the same statement holds for $d_{M}(X), d_{M * v}(X)$, and $d_{M+v}(X)$.

We may state the max analog of Theorem [5.5, using the duality $\max (M) * V=$ $\min (M * V)$ - note again the change from $M \bar{*} v$ to $M+v$.

Theorem 5.6. Let $M$ be a delta-matroid, and $v \in V$. Then the equicardinal set systems $\max (M), \max (M * v)$, and $\operatorname{maxc}(M+v)$ are such that precisely two of the three are equal, to say $M_{1}$. Moreover, the third $M_{2}$ is such that $d_{M_{1}}=d_{M_{2}}+1$ and $M_{2} \widehat{\backslash} v=M_{1}$.

Proof. We have $\max (M)=\min (M * V) * V, \max (M * v)=\min ((M * V) * v) * V$, and $\max (M+v)=\min (M+v * V) * V=\min ((M * V) \bar{*} v) * V$. Now apply Theorem 5.5 to delta-matroid $M * V$. Finally, let $M_{1}=M_{1}^{\prime} * V$ and $M_{2}=M_{2}^{\prime} * V$, where $M_{1}^{\prime}$ and $M_{2}^{\prime}$ are the two set systems of Theorem 5.5 belonging to delta-matroid $M * V$. We

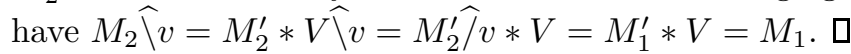

Of course, $\max (M)$ and $\max (M * v)$ are matroids. If $M+v$ is a delta-matroid, then $\max (M+v)=\operatorname{maxc}(M+v)$ is also a matroid. The matroid formulation of Theorem 5.6 for the case where $M+v$ is a delta-matroid is given in the Introduction.

Note that $d_{M_{1}}=d_{M_{2}}+1$ means that the cardinality of the sets in $M_{2}$ is exactly one smaller than the cardinality of the sets in $M_{1}$.

Note also that the definition of loop complementation may be formulated through the distance measure since $d_{M+v}(X)=0$ iff $X \in M+v$. We have therefore, for $X \subseteq V$, by definition of loop complementation, $d_{M+v}(X)=0$ iff $d_{M}(X)=0$ when $v \notin X$, and $d_{M+v}(X)=0$ iff $\left(d_{M}(X)=0\right) \oplus\left(d_{M * v}(X)=0\right)$ when $v \in X$. Recall that $d_{M+v}(X)=d_{M}(X)$, hence the case $v \notin X$ is extended to arbitrary values of $d_{M+v}(X)$. Moreover, by Theorem 5.6, the case $v \in X$ is extended for delta-matroids to arbitrary values of $d_{M+v}(X)$ through the $m, m, m+1$ property (by extending $\oplus$ in a suitable way from Booleans to integers).

One may wonder whether or not the property of Theorem 5.5 characterizes deltamatroids. The next example illustrates that this is not the case.

ExAmple 5.1. Let $M=(V,\{\varnothing, V\})$. Recall that $M$ is not a delta-matroid for $|V| \geq 3$. Assume now that $|V| \geq 3$ is even. Let $X \subseteq V$ and $v \in X$. As the (two) sets in $M$ are of equal parity, the distances $d_{M}(X)$ and $d_{M * v}(X)=d_{M}(X \Delta\{v\})$ are of different parity (as $|X|$ and $|X \Delta\{v\}|$ are of different parity). Now, by Lemma 3.2, $d_{M+v}(X)=\min \left\{d_{M}(X), d_{M * v}(X)\right\}$, and we have that $d_{M}(X), d_{M * v}(X)$, and $d_{M+v}(X)$ are, in this order, either of the form $m, m+1$, and $m$, or of the form $m+1, m$, and $m$.

6. Representable Delta-Matroids. In this section we consider the case where a delta-matroid $M$ is represented by a matrix $A$. We show that in that case the notion of distance to $X$ in the represented delta-matroid closely matches that of nullity of the principal submatrix $A[X]$.

For a $V \times V$ matrix $A$ (the columns and rows of $A$ are indexed by finite set $V$ ) and $X \subseteq V, A[X]$ denotes the principal submatrix of $A$ with respect to $X$, i.e., the $X \times X$ matrix obtained from $A$ by restricting to rows and columns in $X$. We also define $A \backslash X=A[V \backslash X]$. For $V \times V$ matrix $A$ we consider the associated set system $\mathcal{M}_{A}=\left(V, D_{A}\right)$ with $D_{A}=\{X \subseteq V \mid A[X]$ is nonsingular $\}$. Observe that $\mathcal{M}_{A[X]}=\mathcal{M}_{A}[X]$, and $\mathcal{M}_{A \backslash X}=\mathcal{M}_{A} \backslash X$.

It is shown in 4 that $\mathcal{M}_{A}$ is a delta-matroid when $A$ is symmetric or skewsymmetric (over some field $\mathbb{F}$ ). Note that $\varnothing \in \mathcal{M}_{A}$. We say that delta-matroid $M$ is 
representable over $\mathbb{F}$ if $M=\mathcal{M}_{A} * X$ for some skew-symmetric matrix $A$ and $X \subseteq V$; $A$ is called a representation of $M$. A delta-matroid is called binary if it is representable over $\mathbb{F}_{2}$.

Recall that for a $W \times V$ matrix $A$, the column matroid $N=(V, B)$ of $A$ described by its bases is such that, for $X \subseteq V, X \in B$ iff the columns of $A$ belonging to $X$ form a basis of the column space of $A$. Matrix $A$ is said to represent $N$. For matroids, this usual sense of representability coincides with representability in the delta-matroid sense, see 4.4 of [4. Hence, every binary matroid is a binary delta-matroid.

We now formulate the matroid version of the strong principal minor theorem [18 (the original result is more general, as it considers quasi-symmetric matrices over a division ring), see also [10, Lemma 10].

Proposition 6.1 (Strong Principal Minor Theorem [18). Let $A$ be $a V \times V$ symmetric or skew-symmetric matrix (over some field). Then $\max \left(\mathcal{M}_{A}\right)$ equals the column matroid of $A$ (described by its bases).

As a consequence of the strong principal minor theorem, the sets in $\max \left(\mathcal{M}_{A}\right)$ are all of cardinality equal to the $\operatorname{rank} r(A)$ of $A$ - this fact is known as the principal minor theorem. We now use the principal minor theorem to obtain that the distance $X \subseteq V$ to $\mathcal{M}_{A}$ corresponds to the nullity of $A[X]$. We will also use the strong principal minor theorem to prove Theorem 7.1

TheOREm 6.2. Let $A$ be $a V \times V$ symmetric or skew-symmetric matrix (over some field). Then $d_{\mathcal{M}_{A}}(X)=n(A[X])$ for every $X \subseteq V$.

Proof. By Theorem [5.2, $d_{\mathcal{M}_{A}}(X)=d_{\mathcal{M}_{A}[X]}(X)$ (note that $\mathcal{M}_{A}[X]$ is proper as $\left.\varnothing \in \mathcal{M}_{A}\right)$. Now, $d_{\mathcal{M}_{A}[X]}(X)=|X|-|Z|$ with $Z \in \max \left(\mathcal{M}_{A}[X]\right)$. Moreover, $\mathcal{M}_{A}[X]=\mathcal{M}_{A[X]}$. By the principal minor theorem, $|Z|=r\left(\mathcal{M}_{A[X]}\right)=r(A[X])$, and so $d_{\mathcal{M}_{A[X]}}(X)=n(A[X])$.

To extend the notion of nullity to delta-matroids (or proper set systems in general), we regard $d_{M}(X)$ as the nullity of $X$ in $M$. We may now also define the rank $r_{M}(X)$ of $X$ in $M$ by $r_{M}(X)=|X|-d_{M}(X)$. In this way we have $r_{\mathcal{M}_{A}}(X)=r(A[X])$, where $r(A[X])$ denotes the rank of matrix $A[X]$.

REMARK 6.1. There have been a number of rank functions introduced for deltamatroids. In [8] the rank of $X \subseteq V$ is defined as $r_{M}^{\prime}(X)=\max \{|X \cap Y|+\mid(V \backslash$ $X) \cap(V \backslash Y)|| Y \in M\}$. It is easy to verify that $r_{M}^{\prime}(X)=\max \{|V \backslash(X \Delta Y)| \mid$ $Y \in M\}=|V|-\min \{|X \Delta Y| \mid Y \in M\}$. Therefore, $r_{M}^{\prime}(X)=|V|-d_{M}(X)$ and hence the notion is slightly different from the rank function $r_{M}(X)$ defined in this paper. Also, in [5] the birank of $X, Y \subseteq V$ with $X \cap Y=\varnothing$ is defined as $r_{M}^{\prime \prime}(X, Y)=\max \{|Z \cap X|+|(V \backslash Z) \cap Y| \mid Z \in M\}$. We have $r_{M}^{\prime \prime}(X, V \backslash X)=r_{M}^{\prime}(X)$. Finally, in [17] the birank of $X, Y \subseteq V$ with $X \cap Y=\varnothing$ is defined as $r_{M}^{\prime \prime \prime}(X, Y)=$ $\max \{|Z \cap X|-|Z \cap Y| \mid Z \in M\}$. Function $r_{M}^{\prime \prime \prime}(X, Y)$ does correspond to the rank $r_{M}(X)$ as defined in this paper, as $r_{M}^{\prime \prime \prime}(X, V \backslash X)=r_{M}(X)$. However none of these papers on delta-matroids (explicitly) considers nullity as a distance measure.

A delta-matroid $M$ is called even if the cardinality of the sets in $M$ all have equal parity. Let $A$ be a skew-symmetric matrix over some field $\mathbb{F}$. It easily follows from 4 , Thm 4.3.3] that delta-matroid $\mathcal{M}_{A}$ is even when $A$ is zero-diagonal (note that this condition is only relevant when $\mathbb{F}$ is of characteristic 2 ). We now obtain the following corollary to Theorem 6.2.

Corollary 6.3. Let $A$ be $a V \times V$ zero-diagonal skew-symmetric matrix, and $v \in V$. Then $n(A)$ and $n(A \backslash v)$ differ by precisely 1 .

Proof. Since the cardinality of the sets in $\mathcal{M}_{A}$ have a common parity, for all $X \subseteq$ $V, d_{\mathcal{M}_{A}}(X)$ is odd iff $d_{\mathcal{M}_{A}}(X \Delta\{v\})$ is even. As $\left|d_{\mathcal{M}_{A}}(X)-d_{\mathcal{M}_{A}}(X \Delta\{v\})\right| \leq 1$, we 
have $\left|d_{\mathcal{M}_{A}}(X)-d_{\mathcal{M}_{A}}(X \Delta\{v\})\right|=1$. Let now $X=V$ and we obtain by Theorem6.2. $|n(A)-n(A \backslash v)|=1$.

7. Application: Graphs. In this section we translate the above results to the realm of graphs, where, e.g., the operations $+v$ and $* v$ have their own specific interpretation. We consider undirected graphs without parallel edges, but we do allow loops. For a graph $G=(V, E)$ and $x \in V$, we have $\{x\} \in E$ iff $x$ has a loop. With a graph $G$ one associates its adjacency matrix $A(G)$, which is a $V \times V$ matrix $\left(a_{u, v}\right)$ over $\mathbb{F}_{2}$ with $a_{u, v}=1$ iff $\{u, v\} \in E$ (with possibly $u=v$ ). In this way, the family of graphs with vertex set $V$ corresponds precisely to the family of symmetric $V \times V$ matrices over $\mathbb{F}_{2}$. Therefore we often make no distinction between a graph and its matrix, so, e.g., by the null space (or kernel) and nullity (i.e., dimension of the null space) of graph $G$, denoted by $\operatorname{ker}(G)$ and $n(G)$ respectively, we mean the null space and nullity of its adjacency matrix $A(G)$ (computed over $\mathbb{F}_{2}$ ). Also, for $X \subseteq V, G[X]=A(G)[X]$ is the subgraph of $G$ induced by $X$. By convention, the empty graph/matrix is nonsingular. Similar as for set systems, we often write $V$ to denote the vertex set of the graph under consideration.

For a graph $G$ and a set $X \subseteq V$, the graph obtained after loop complementation for $X$ on $G$, denoted by $G+X$, is obtained from $G$ by adding loops to vertices $v \in X$ when $v$ does not have a loop in $G$, and by removing loops from vertices $v \in X$ when $v$ has a loop in $G$. Hence, if one considers a graph as a matrix, then $G+X$ is obtained from $G$ by adding the $V \times V$ matrix with elements $x_{i, j}$ such that $x_{i, j}=1$ if $i=j \in X$ and 0 otherwise. Note that $(G+X)+Y=G+(X \Delta Y)$.

Given the set system $\mathcal{M}_{G}=\mathcal{M}_{A(G)}=\left(V, D_{G}\right)$ for some graph $G=(V, E)$, one can (re)construct the graph $G$, see [7, Property 3.1]. Hence the function $\mathcal{M}_{(\cdot)}$ which assigns to each graph $G$ its set system $\mathcal{M}_{G}$ is injective. In this way, the family of graphs (with set $V$ of vertices) can be considered as a subset of the family of set systems (over set $V$ ). Note that $\mathcal{M}_{(\text {.) }}$ is not injective for matrices over $\mathbb{F}_{2}$ in general: e.g., for fixed $V$ with $|V|=2$, the $2 \times 2$ zero matrix and the matrix $\left(\begin{array}{cc}0 & 1 \\ 0 & 0\end{array}\right)$ correspond to the same set system.

It is shown in [11] that $\mathcal{M}_{G+X}=\mathcal{M}_{G}+X$ for any graph $G$ and $X \subseteq V$. Therefore the operation $+X$ on set systems $M$ is a generalization of loop complementation on graphs $G$ - which explains its name.

If $G$ is a graph and $u$ a vertex 1 of $G$, then the result of local complementation of $u$ on $G$, denoted by $\operatorname{loc}_{u}(G)$, is the graph obtained from $G$ by "toggling" the edges in the neighbourhood $N_{G}(u)=\{v \in V \mid\{u, v\} \in E(G), u \neq v\}$ of $u$ in $G$ : for each $v, w \in N_{G}(u),\{v, w\} \in E(G)$ iff $\{v, w\} \notin E\left(\operatorname{loc}_{u}(G)\right)$ (again, $v=w$ is possible). The other edges are left unchanged.

If $u$ is a looped vertex of $G$, then it is shown in [14] that $\mathcal{M}_{G} * u=\mathcal{M}_{\operatorname{loc}_{u}(G)}$. Moreover, if $u$ is a unlooped vertex of $G$, then $\mathcal{M}_{G} \bar{*} u=\mathcal{M}_{\operatorname{loc}_{u}(G)}$ (see [11]). In this way, local complementation is defined for delta-matroids. For convenience, we define the pivot of a looped vertex $u$ on $G$, denoted as $G * u$, by $\operatorname{loc}_{u}(G)$ (it is not defined on unlooped vertices). Similarly, we define the dual pivot of an unlooped vertex $u$ on $G$, denoted as $G \bar{*} u$, by $\operatorname{loc}_{u}(G)$ (it is not defined on looped vertices). Thus, if $u$ is looped, then $\mathcal{M}_{G} * u=\mathcal{M}_{G * u}$, and if $u$ is unlooped, then $\mathcal{M}_{G} \bar{*} u=\mathcal{M}_{G \bar{*} u}$. In general, for a set $X \in \mathcal{M}_{G}, \mathcal{M}_{G} * X=\mathcal{M}_{G * X}$ where $G * X$ is a graph called the

\footnotetext{
${ }^{1}$ Local complementation is often defined on simple graphs; here we consider the obvious extension to graphs where loops are allowed. Note that local complementation may be applied here to a nonlooped vertex $u$, which is different from, e.g., [11].
} 
pivot (or principal pivot transform) [23] of $G$ on $X$ [4] - clearly, $\mathcal{M}_{G} * X$ does not correspond to a graph if $X \notin \mathcal{M}_{G}$.

As usual, a vector $v$ indexed by $V$ over $\mathbb{F}_{2}$ may be identified by a subset $X \subseteq V$, where $v$ at position $x \in V$ is 1 iff $x \in X$. In this way, we regard $\operatorname{ker}(G)$ for a graph $G$ as a subset of $2^{V}$. By the strong principal minor theorem we have that the cycle space of the matroid $\max \left(\mathcal{M}_{G}\right)$ is precisely $\operatorname{ker}(G)$. Of course, the nullity of the matroid $\max \left(\mathcal{M}_{G}\right)$ is the dimension $\operatorname{dim}(\operatorname{ker}(G))$ of $\operatorname{ker}(G)$.

If we restrict now Theorem 5.6 for the case where the delta-matroid $M$ is equal to $\mathcal{M}_{G}$, then we obtain the following result.

THEOREM 7.1. Let $G$ be a graph having a looped vertex $v$. Then $\operatorname{ker}(G), \operatorname{ker}(G * v)$, and $\operatorname{ker}(G+v)$ are such that precisely two of the three are equal, to say $K_{1}$, and the third, $K_{2}$, is such that $\operatorname{dim}\left(K_{2}\right)=\operatorname{dim}\left(K_{1}\right)+1$ and $K_{1}=\left\{X \in K_{2} \mid v \notin X\right\}$.

Proof. From Theorem 6.2 we know that for any graph $G^{\prime}$ the dimension of the kernel equals $n\left(G^{\prime}\right)=d_{\mathcal{M}_{G^{\prime}}}(V)$, i.e., the cardinality of sets in $\min \left(\mathcal{M}_{G^{\prime}} * V\right)$ which are complements of sets in $\max \left(\mathcal{M}_{G^{\prime}}\right)$.

By Theorem [5.6] $\max \left(\mathcal{M}_{G}\right), \max \left(\mathcal{M}_{G * v}\right)$, and $\max \left(\mathcal{M}_{G+v}\right)$ are such that precisely two of the three are equal, to say $M_{1}$, and the nullity of the third, $M_{2}$, is one larger than the nullity of $M_{1}$. Moreover, the family of circuits of $M_{1}$ is obtained from the family of circuits of $M_{2}$ by removing the sets containing $v$.

Hence (by discussion above), $\operatorname{ker}(G), \operatorname{ker}(G * v)$, and $\operatorname{ker}(G+v)$ are such that precisely two of the three are equal, to say $K_{1}$. The third, $K_{2}$, is such that $\operatorname{dim}\left(K_{2}\right)=$ $\operatorname{dim}\left(K_{1}\right)+1$ and $K_{1}=\left\{X \in K_{2} \mid v \notin X\right\}$.

Theorem 7.1 is similar to a result of Traldi 22, Lemma 23], where graph $G^{\prime}$, obtained from $G$ by removing all edges incident to $v$ except for the loop on $v$, is considered instead of $G * v$. Moreover, Theorem 7.1] is essentially [3, Theorem (9.4)] for the case where $G$ is a fundamental graph of an isotropic system.

Let $G$ be a graph with looped vertex $v$. By Theorem 7.1 the values of $n(G)$, $n(G * v)$, and $n(G+v)$ are such that precisely two of the three are equal, to say $m$, and the third is equal to $m+1$. It is shown in [10] that the adjacency matrix of $G * v \backslash v$ is the Schur complement of $v$ on the adjacency matrix of $G$, and moreover it is well known, see e.g. [26], that the Schur complement retains the nullity, i.e., $n(G * v \backslash v)=n(G)$. Hence, we have $n(G * v)=n(G \backslash v)$ and we obtain as a consequence of Theorem 7.1 the following result of [1].

Proposition 7.2 (Lemma 2 of [1]). Let $G$ be a graph and $v \in V$. Then the values of $n(G), n(G \backslash v)$, and $n(G+v)$ are such that precisely two of the three are equal, to say $m$, and the third is equal to $m+1$.

Corollary 6.3 for the case where the field is $\mathbb{F}_{2}$ may be stated as follows.

Corollary 7.3. Let $G$ be a graph, and $v \in V$ a vertex of $G$. If $G$ has no loops, then $n(G)$ and $n(G \backslash v)$ differ by precisely 1.

8. Vertex-Flip-Safe Delta-Matroids. Recall from Section 5 that the result of applying dual pivot or loop complementation on a delta-matroid is not necessarily a delta-matroid. In this section we consider families of delta-matroids that are closed under invertible vertex flips in general. In particular, we show that the binary deltamatroids form one such family. As a consequence, for binary delta-matroid $M$ the set systems $M * v$ and $M \bar{*} v$ in Theorem 5.5 are binary delta-matroids.

Definition 8.1. Let $M$ be a delta-matroid. We say that $M$ is a vertex-flip-safe (or vf-safe for short) if for any sequence $\varphi$ of invertible vertex flips (equivalently, pivots and loop complementations) over $V$ we have that $M \varphi$ is a delta-matroid. 
Hence, $M$ is a vf-safe delta-matroid iff each set system in the orbit of $M$ under pivot and loop complementation is a delta-matroid.

We say that a family of delta-matroids is vf-closed if the family is closed under invertible vertex flips. We now show that the family of binary delta-matroids is vfclosed. First we remark that this is not trivial. While we know that for a graph $G$ and $X \subseteq V,(1) \mathcal{M}_{G} * X$ is a binary delta-matroid by definition, and (2) $\mathcal{M}_{G}+X=\mathcal{M}_{G+X}$ corresponds to a graph, it is not immediately clear that, e.g., $\mathcal{M}_{G} * X+Y$ is a deltamatroid for all $Y \subseteq V$ (recall that $\mathcal{M}_{G} * X$ does not correspond to a graph when $\left.X \notin \mathcal{M}_{G}\right)$.

THEOREM 8.2. The family of binary delta-matroids is vf-closed. In particular, every binary delta-matroid is vf-safe.

Proof. Let $M$ be a binary delta-matroid. Hence $M$ is of the form $\mathcal{M}_{G} * X$ for some graph $G$ and $X \subseteq V$. Let $\varphi$ be a sequence of invertible vertex flips over $V$. Let $W \in \mathcal{M}_{G} * X \varphi$, and consider now $\varphi^{\prime}=* X \varphi * W$. By [11, Corollary 15], $\varphi^{\prime}$ can be put in the following normal form: $\mathcal{M}_{G} \varphi^{\prime}=\mathcal{M}_{G}+Z_{1} * Z_{2}+Z_{3}$ for some $Z_{1}, Z_{2}, Z_{3} \subseteq V$ with $Z_{1} \subseteq Z_{2}$. We have $\mathcal{M}_{G}+Z_{1}=\mathcal{M}_{G+Z_{1}}$. Thus $\mathcal{M}_{G}+Z_{1} * Z_{2}+Z_{3}=\mathcal{M}_{G+Z_{1}} * Z_{2}+Z_{3}$. By construction $\varnothing \in \mathcal{M}_{G} \varphi^{\prime}$. Hence we have $\varnothing \in \mathcal{M}_{G+Z_{1}} * Z_{2}$. Therefore $Z_{2} \in \mathcal{M}_{G+Z_{1}}$ and so $G+Z_{1} * Z_{2}$ is defined. Consequently, $G^{\prime}=G+Z_{1} * Z_{2}+Z_{3}$ is defined and $\mathcal{M}_{G} \varphi^{\prime}=\mathcal{M}_{G^{\prime}}$. Hence $M \varphi=\mathcal{M}_{G} * X \varphi=\mathcal{M}_{G^{\prime}} * W$ and thus $G^{\prime}$ represents $M \varphi$. Consequently, $M \varphi$ is a binary delta-matroid.

We consider some specific matroids to illustrate the scope of this notion. By Theorem 8.2 every binary matroid is vf-safe. Not every matroid is a vf-safe matroid. The 6-point line, i.e., $U_{2,6}=(V,\{\{u, v\} \mid u, v \in V, u \neq v\})$ with $|V|=6$, is not vf-safe. Recall that $X \in M+V$ iff the number of sets in $M[X]$ is odd. We have $V \in U_{2,6}+V$ as the number of sets in $U_{2,6}$ is $\left(\begin{array}{c}6 \\ 2\end{array}\right)=15$ (odd), while the sets of cardinality 4 and 5 are not in $U_{2,6}+V$ as $\left(\begin{array}{l}5 \\ 2\end{array}\right)=10$ and $\left(\begin{array}{l}4 \\ 2\end{array}\right)=6$ are even. Consequently, the symmetric exchange axiom does not hold for $V \in U_{2,6}+V$ (as neither $V \backslash\{u\} \in U_{2,6}+V$ nor $V \backslash\{u, v\} \in U_{2,6}+V$ for any $\left.u, v \in V\right)$.

Based on Theorem 4.2 one easily verifies (by computer) that several small (nonbinary) matroids are vf-safe. Such examples include the matroids $U_{2,4}, U_{2,5}, U_{3,6}, Q_{6}$, $\mathcal{W}^{3}, P_{8}, P_{8}^{=}$, and Pappus. For information on these matroids, see the Appendix on interesting matroids in 20.

We turn to minors. A minor of a delta-matroid $M$ is a proper set system obtained from $M$ by any sequence of $\backslash v$ (deletion) and $* v \backslash v$ (contraction) operations. A minor of $M$ is thus a proper set system of the form $M * X \backslash Y$ with $X \subseteq Y \subseteq V$. Consequently, a minor of a delta-matroid is again a delta-matroid. Also note that this notion of minor restricted to matroids coincides with the usual notion of minor for matroids.

THEOREM 8.3. The family of vf-safe delta-matroids is minor-closed. In particular, the family of vf-safe matroids is minor-closed.

Proof. It suffices to consider $M \backslash u$ for a vf-safe delta-matroid and some $u \in V$. Let $\varphi$ be a sequence of invertible vertex flips on $V \backslash\{u\}$. Then $M^{\prime}=(M \backslash u) \varphi=(M \varphi) \backslash u$. Moreover, $M \varphi$ is a delta-matroid as $M$ is a delta-matroid. Also, $M^{\prime}$ is proper, as $M \backslash u$ is proper. Consequently, $M^{\prime}=(M \varphi) \backslash u$ is a delta-matroid.

Theorem 8.3 suggests looking for an excluded-minor characterization for the class of vf-safe matroids. By computer we found that the matroids $U_{2,6}, U_{4,6}, P_{6}, F_{7}^{-}$, and $\left(F_{7}^{-}\right)^{*}$ (see again [20] for a description of these matroids) are excluded minors for the family of vf-safe matroids. Moreover, using the database of D. Mayhew and G.F. Royle [19, we confirmed that these are the only excluded minors with 9 or less 
elements. We notice similarity with the excluded-minor characterization of quaternary matroids (i.e., the matroids representable over $G F(4)$ ) [15], where it is shown that a matroid $M$ is quaternary iff no minor of $M$ is isomorphic to $U_{2,6}, U_{4,6}, P_{6}, F_{7}^{-}$, $\left(F_{7}^{-}\right)^{*}, P_{8}$, or $P_{8}^{=}$. Hence, we conjecture the following (which consequently has been verified for matroids with 9 or less elements).

CONJECTURe 8.1. Every quaternary matroid is vf-safe.

Let $\mathcal{N}$ be the family of matroids that have no minors isomorphic to $U_{2,6}, U_{4,6}$, $P_{6}, F_{7}^{-}$, or $\left(F_{7}^{-}\right)^{*}$. It is shown in [16, Corollary 1.2] that $\mathcal{N}$ can be constructed by taking direct sums and 2 -sums of copies of $P_{8}^{=}$, minors of $S(5,6,12)$, and quaternary matroids (see again [20] for a description of $S(5,6,12)$ ). In this light, we have verified (by computer and using internal symmetries of the matroid) and found that $S(5,6,12)$ is vf-safe as well. Hence we conjecture that the above list of excluded minors for the class of vf-safe matroids is complete.

We finally note that not every quaternary delta-matroid is vf-safe. For example, the non-vf-safe delta-matroid $\left(V, 2^{V} \backslash\{\{u\}\}\right)$ with $V=\{u, v, w\}$ (this delta-matroid differs from the non-vf-safe delta-matroid of Section 5 by a pivot) is represented by the following skew-symmetric matrix over $G F(4)$ :

$$
\begin{aligned}
& \begin{array}{lll}
u & v & w
\end{array} \\
& \begin{array}{l}
u \\
w \\
w
\end{array}\left(\begin{array}{lll}
0 & a & b \\
a & 1 & 0 \\
b & 0 & 1
\end{array}\right)
\end{aligned}
$$

where $a$ and $b$ are the two elements distinct from 0 and 1 in $G F(4)$.

Acknowledgements. We thank Lorenzo Traldi for a stimulating correspondence. We are much indebted to the anonymous reviewers for valuable comments on earlier versions of this paper. We thank Gordon Royle for kindly sending to us the database of matroids with nine elements from [19. R.B. was supported by the Netherlands Organization for Scientific Research (NWO), project "Annotated graph mining".

\section{REFERENCES}

[1] P. Balister, B. Bollobás, J. Cutler, And L. Pebody, The interlace polynomial of graphs at -1, European Journal of Combinatorics, 23 (2002), pp. 761-767.

[2] A.V. Borovik, I. Gelfand, And N. White, Symplectic matroids, Journal of Algebraic Combinatorics, 8 (1998), pp. 235-252.

[3] A. Bouchet, Isotropic systems, European Journal of Combinatorics, 8 (1987), pp. 231-244.

[4] - Representability of $\Delta$-matroids, in Proc. 6th Hungarian Colloquium of Combinatorics, Colloquia Mathematica Societatis János Bolyai, vol. 52, North-Holland, 1987, pp. 167-182.

[5] —, Maps and $\Delta$-matroids, Discrete Mathematics, 78 (1989), pp. 59-71.

[6] - Coverings and delta-coverings, in IPCO, E. Balas and J. Clausen, eds., vol. 920 of Lecture Notes in Computer Science, Springer, 1995, pp. 228-243.

[7] A. Bouchet and A. Duchamp, Representability of $\Delta$-matroids over GF(2), Linear Algebra and its Applications, 146 (1991), pp. 67-78.

[8] A. Bouchet and B. Jackson, Parity systems and the delta-matroid intersection problem, Electronic Journal of Combinatorics, 7 (2000).

[9] R. Brijder And H.J. Hoogeboom, Interlace polynomials for delta-matroids. [arXiv:1010.4678], 2010.

[10] - Maximal pivots on graphs with an application to gene assembly, Discrete Applied Mathematics, 158 (2010), pp. 1977-1985.

[11] — The group structure of pivot and loop complementation on graphs and set systems, European Journal of Combinatorics, 32 (2011), pp. 1353-1367. 
[12] R.A. Brualdi, Comments on bases in dependence structures, Bulletin of the Australian Mathematical Society, 1 (1969), pp. 161-167.

[13] R. Chandrasekaran and S.N. Kabadi, Pseudomatroids, Discrete Mathematics, 71 (1988), pp. 205-217.

[14] J.F. GeElen, A generalization of Tutte's characterization of totally unimodular matrices, Journal of Combinatorial Theory, Series B, 70 (1997), pp. 101-117.

[15] J.F. Geelen, A. M. H. Gerards, And A. Kapoor, The excluded minors for GF(4)representable matroids, Journal of Combinatorial Theory, Series B, 79 (2000), pp. 247-299.

[16] J.F. Geelen, J.G. Oxley, D. Vertigan, and G.P. Whittle, On the excluded minors for quaternary matroids, Journal of Combinatorial Theory, Series B, 80 (2000), pp. 57-68.

[17] S.N. Kabadi And R. SRIDhaR, $\Delta$-matroid and jump system, Journal of Applied Mathematics and Decision Sciences, 2005 (2005), pp. 95-106.

[18] V. Kodiyalam, T.Y. Lam, and R.G. Swan, Determinantal ideals, Pfaffian ideals, and the principal minor theorem, in Noncommutative Rings, Group Rings, Diagram Algebras and Their Applications, American Mathematical Society, 2008, pp. 35-60.

[19] D. Mayhew and G.F. Royle, Matroids with nine elements, Journal of Combinatorial Theory, Series B, 98 (2008), pp. 415-431.

[20] J.G. OxLEY, Matroid theory, Second Edition, Oxford University Press, 2011.

[21] L. Traldi, Binary nullity, Euler circuits and interlace polynomials, European Journal of Combinatorics, 32 (2011), pp. 944-950.

[22] - On the linear algebra of local complementation, Linear Algebra and its Applications, 436 (2012), pp. 1072-1089.

[23] M.J. Tsatsomeros, Principal pivot transforms: properties and applications, Linear Algebra and its Applications, 307 (2000), pp. 151-165.

[24] A.W. Tucker, A combinatorial equivalence of matrices, in Combinatorial Analysis, Proceedings of Symposia in Applied Mathematics, vol. X, American Mathematical Society, 1960, pp. $129-140$.

[25] D.J.A. Welsh, Matroid theory, Academic Press, 1976.

[26] F. Zhang, The Schur Complement and Its Applications, Springer, 2005. 